\title{
Bouncing Oil Droplet in a Stratified Liquid and its Sudden Death
}

\author{
Yanshen Li, ${ }^{1}$ Christian Diddens, ${ }^{1,2}$ Andrea Prosperetti, ${ }^{1,3}$ Kai Leong Chong, ${ }^{1}$ Xuehua Zhang, ${ }^{4,1, *}$ and Detlef Lohse ${ }^{1,5, \dagger}$ \\ ${ }^{1}$ Physics of Fluids group, Max-Planck Center Twente for Complex Fluid Dynamics, Department of Science and Technology, \\ Mesa+Institute, and J. M. Burgers Centre for Fluid Dynamics, University of Twente, P.O. Box 217, 7500 AE Enschede, Netherlands \\ ${ }^{2}$ Department of Mechanical Engineering, Eindhoven University of Technology, P.O. Box 513, 5600 MB Eindhoven, Netherlands \\ ${ }^{3}$ Department of Mechanical Engineering, University of Houston, Texas 77204-4006, USA \\ ${ }^{4}$ Department of Chemical and Materials Engineering, University of Alberta, 12-380 Donadeo Innovation Centre for Engineering, \\ Edmonton, T6G1H9 Alberta, Canada \\ ${ }^{5}$ Max Planck Institute for Dynamics and Self-Organization, 37077 Göttingen, Germany
}

(Received 27 November 2018; revised manuscript received 12 February 2019; published 17 April 2019)

\begin{abstract}
Droplets can self-propel when immersed in another liquid in which a concentration gradient is present. Here we report the experimental and numerical study of a self-propelling oil droplet in a vertically stratified ethanol-water mixture: At first, the droplet sinks slowly due to gravity, but then, before having reached its density matched position, jumps up suddenly. More remarkably, the droplet bounces repeatedly with an ever increasing jumping distance, until all of a sudden it stops after about $30 \mathrm{~min}$. We identify the Marangoni stress at the droplet-liquid interface as responsible for the jumping: its strength grows exponentially because it pulls down ethanol-rich liquid, which in turn increases its strength even more. The jumping process can repeat because gravity restores the system. Finally, the sudden death of the jumping droplet is also explained. Our findings have demonstrated a type of prominent droplet bouncing inside a continuous medium with no wall or sharp interface.
\end{abstract}

DOI: 10.1103/PhysRevLett.122.154502

Swimming droplets [1] are droplets that self-propel when immersed in another liquid. They are of great importance for their relevance to (bio)chemical reactors $[2,3]$. They also serve as a model system for studying collective behavior in biological populations [4-8]. One of the fundamental mechanisms leading to their selfpropulsion is the so-called Marangoni effect $[9,10]$. It is induced by the nonuniform interfacial tension of the droplet that can be generated by chemical reactions [11-15], solubilization [1,16-20], phase separation [21-25], or by a global temperature or solute gradient [26]. The present work focuses on the last type, which is commonly encountered in nature [27].

A major focus of earlier studies was on the dynamics of swimming droplets [20]. The motion of a droplet in a global solute gradient is believed to be governed by the competition of droplet speed and the diffusivity of the background concentration field, which is characterized by the Péclet number Pe, which is the ratio between the diffusive and the inertial time scale. Previous works identified two regimes of droplet motion based on the framework of diffusiophoresis $[10,28,29]$ : For small Pe, the concentration gradient is not affected by fluid motion and thus the droplet movement can persist. In contrast, for large Pe, the sharp concentration gradient at the periphery of the droplet is always smoothed out, and thus the motion of the droplet is slowed down [30]. However, in this Letter, we conduct experiments and simulations to demonstrate that when combined with gravity, the Marangoni stress on an oil droplet in a stratified ethanol-water mixture can oscillate between large and small $\mathrm{Pe}$, leading to a continuous bouncing of the droplet. And, more surprisingly, the amplitude of the droplet oscillatory motion even increases before it suddenly stops. Contrary to the commonly held concept that droplet bouncing requires a wall [31-33] or a sharp interface [34]; here the droplet bounces in the bulk of a continuous medium, which only requires a large enough concentration gradient.

In the experiment, $1.8 \mathrm{~mL}$ ethanol is carefully injected into a cuvette $(10 \times 10 \times 45 \mathrm{~mm})$ containing $1.8 \mathrm{~mL}$ water to produce a vertical density stratification. Then a $0.5 \mu \mathrm{L}$ $(R=0.44 \mathrm{~mm})$ oil drop of trans-Anethole is released in it. The motion of the droplet is visualized with a Nikon camera aiming from the side. A series of typical snapshots of the droplet motion within the first two jumping cycles are shown in Fig. 1(a). The height of the droplet center $h(t)$ is plotted over the first two cycles in Fig. 1(c) and over the entire jumping lifetime in Fig. 1(d), with its final value being taken as 0 . The ethanol fraction $w_{\mathrm{e}}$ as a function of height $h$ is measured by laser deflection and shown in Fig. 1(b). The error bar of $w_{e}$ is within $\pm 2 \mathrm{wt} \%$ (see Supplemental Material [35] for more details.)

The oil droplet has a density of $988 \mathrm{~kg} / \mathrm{m}^{3}$ at $25^{\circ} \mathrm{C}$, which is slightly lighter than water $\left(997 \mathrm{~kg} / \mathrm{m}^{3}\right)$ but much denser than ethanol $\left(785 \mathrm{~kg} / \mathrm{m}^{3}\right)$, so it first sinks slowly due to gravity [Fig. 1(a), (1)-3]. At $59 \mathrm{~s}$ (4), the droplet reaches the height with surrounding mixture density 
(a)

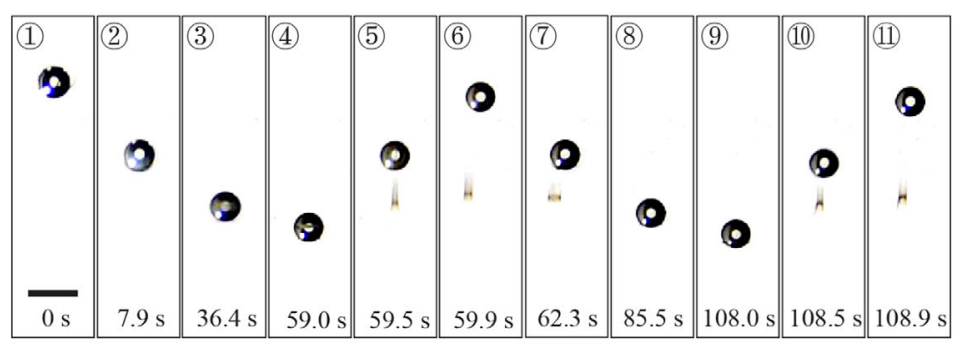

(c)

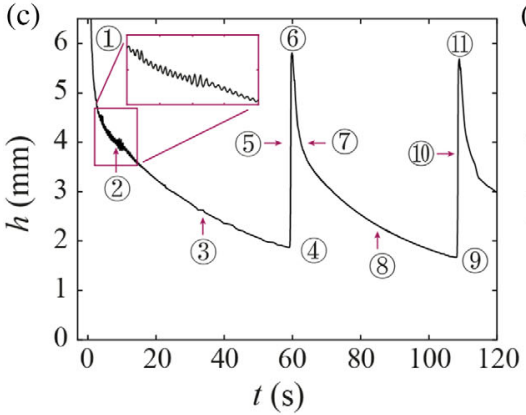

(d)

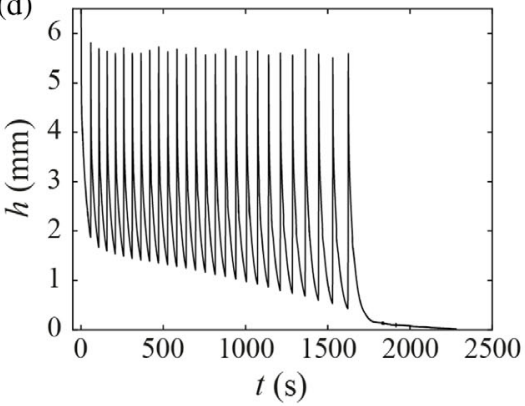

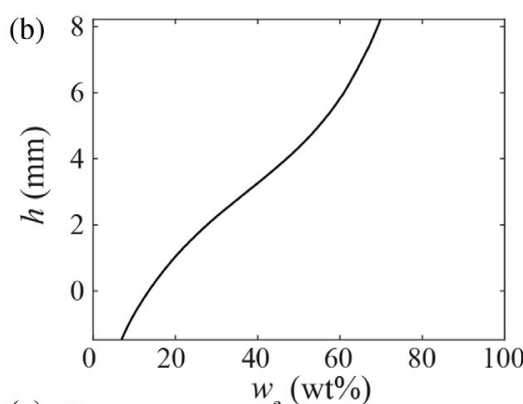

(e)

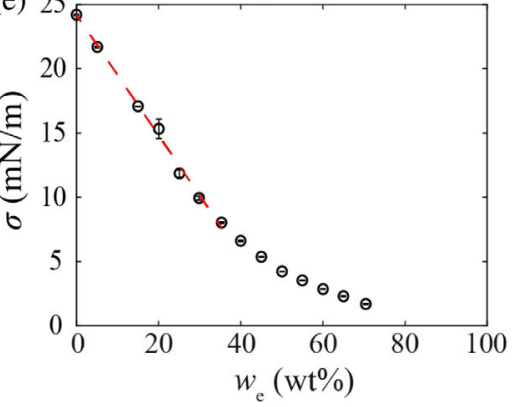

FIG. 1. Continuous jumps and the eventual sudden death of the oil drop. (a) Successive snapshots of the $0.5 \mu \mathrm{L}$ oil drop for the first two cycles. The scale bar is $1 \mathrm{~mm}$. (b) Ethanol weight fraction $w_{e}$ as a function of height $h$ is measured by laser deflection. Error of $w_{e}$ measurement is $\pm 2 \mathrm{wt} \%$. (c) The oil drop's center position $h$ versus time $t$ for the first 2 cycles, with the final height being taken as 0 . The inset shows the vertical oscillation, with frequency $2.15 \mathrm{~Hz}$, during the sinking of the drop. (d) The oil drop's center position $h$ for all the cycles of the jumping process. After each jump, the drop sinks to a lower position, but at each jump, it still reaches almost the same height $h \approx 6 \mathrm{~mm}$, thus even increasing the jumping amplitude. This particular drop jumps 26 times within 30 min. (e) The interfacial tension between oil and ethanol-water mixture. Error bars are the standard deviation over 5 measurements.

$\rho_{\text {mix }}=958 \mathrm{~kg} / \mathrm{m}^{3}\left(w_{\mathrm{e}}=26.6 \mathrm{wt} \%\right)$, which is still lighter than the oil droplet. Surprisingly, instead of sinking continuously, the drop suddenly changes direction and jumps up by $\sim 4 \mathrm{~mm}$ (4-(6), which is more than 4 times its diameter. It reaches the highest position within $0.9 \mathrm{~s}$ (๑), then sinks again for another $49 \mathrm{~s}$ (6-(9). Before reaching the density matched position $(h=0 \mathrm{~mm}$, $\rho_{\text {mix }}=978 \mathrm{~kg} / \mathrm{m}^{3}$ ) again, the drop suddenly jumps up from $108 \mathrm{~s}\left(9, w_{\mathrm{e}}=24.8 \mathrm{wt} \%\right.$ and $\left.\rho_{\text {mix }}=961 \mathrm{~kg} / \mathrm{m}^{3}\right)$ till $108.9 \mathrm{~s}$ (11). It continuously sinks and jumps for another 24 times, then all of a sudden it falls dead after $30 \mathrm{~min}$, as shown in Fig. 1(d). It is noteworthy that by each jump, the drop sinks to a lower position but still returns to almost the same height ( $h \approx 6 \mathrm{~mm}, w_{e} \approx 60 \mathrm{wt} \%$ ); thus the jumping distance increases progressively from 4 to $5.5 \mathrm{~mm}$. We also note that during the sinking of the droplet, there are tiny oscillations as shown in the inset of Fig. 1(c). These oscillations display the Brunt-Väisälä frequency, which describes the vertical oscillation of a fluid parcel in a vertically stratified fluid around its stable position [45-48]. Indeed, the calculated Brunt-Väisälä frequency in our case is $2.08 \mathrm{~Hz}$, which fits well with the observed value $2.15 \mathrm{~Hz}$.

Oil has smaller surface tension with ethanol than with water [Fig. 1(e)], so the ethanol-rich liquid above makes the interfacial tension at the apex of the droplet smaller than that of its bottom. This interfacial tension difference generates a Marangoni flow pointing downwards, which then tends to lift the droplet [1]. The stronger the
Marangoni flow, the faster the droplet will move to the opposite direction. The induced Marangoni flow is essential to the jumping of the droplet, and we confirm this by adding surfactant to the bulk liquid $(0.7 \mathrm{mM}$ sodium dodecyl sulfate, SDS, in both water and ethanol) to suppress the Marangoni stress. With this addition, the droplet only sinks without jumping. This result suggests that during the sinking motion of the droplet, the Marangoni flow is very small.

Apart from the weak Marangoni flow on the droplet induced by the ethanol gradient, it is also settling through a density gradient. As is well known [49-51], a settling particle in a vertically stratified liquid brings lighter liquid down with it, the so called "drift" or "entrainment." Buoyancy of the entrained liquid acts as an extra drag on the droplet, making it sink monotonically towards the density matched position, i.e., no oscillation. Some of the entrained liquid will also go up, forming the "buoyancy jet" [45-48]. The same concept can be adapted to the droplet in our case, except that an extra Marangoni flow, pointing downwards, is superimposed to the flow field of the settling motion. The resulting flow field is then determined by the relative strength of these two effects.

We then perform PIV measurements and shadowgraphy to reveal the flow dynamics during the sinking-jumping process, as shown in the right and left panels of Figs. 2(a)-2(e), respectively. Shadowgraph provides qualitative information on density variations which modulate light intensity. In our 

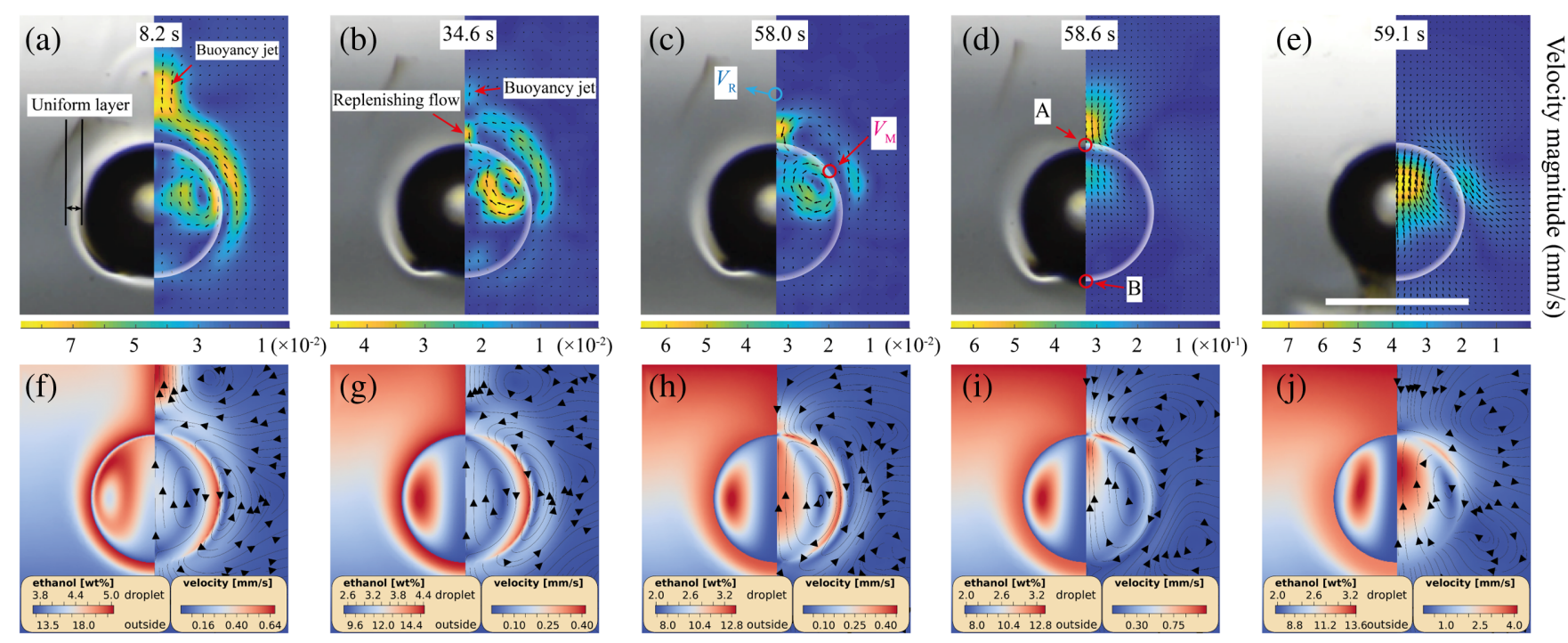

FIG. 2. Shadowgraph (left) and PIV (right) measurements of a $0.5 \mu \mathrm{L}$ oil drop [top row, (a)-(e)] and the numerical simulations [bottom row, (f)-(j)] during the drop's first jump. Light intensity gradient in the background of the shadowgraph indicates the ethanol concentration in the surrounding liquid. Velocities are shown in the laboratory frame. Scale bar is 1 mm, and color bars denote velocity magnitude as well as ethanol fraction. (a),(f) Shortly after release, the buoyant liquid in the droplet's wake generates a relatively strong jet-the buoyancy jet. A drifted uniform layer of ethanol-rich liquid leads to a very weak Marangoni flow. (b), (g) The Marangoni flow becomes relatively strong; thus the flow directly above the drop is pointing downwards; we refer to it as "replenishing flow." Frames (c), (h) are close to the time when the buoyancy jet vanishes. Ethanol-rich liquid above the drop is being brought downwards to its apex, and $V_{M}$ starts to increase. (d),(i) This downward flow brings more ethanol to the apex, further increasing $V_{M}$ in the upper half of the droplet, which then will move as a "puller" [1]. (e),(j) The Marangoni flow has increased by 2 orders of magnitude in less than $1 \mathrm{~s}$, pulling the drop upwards.

case, the ethanol gradient in the surrounding liquid is indicated by light intensity gradient in the background, with brighter regions representing higher ethanol concentration. For the same reason, the drifted layer around the drop in Figs. 2(a)-2(d) is found to have almost uniform light intensity, meaning almost uniform ethanol concentration. The resulting Marangoni flow is very weak $V_{M} \approx$ $0.02 \mathrm{~mm} / \mathrm{s}$ (in droplet reference frame, smaller than the sinking velocity $V_{\text {sink }} \approx 0.04 \mathrm{~mm} / \mathrm{s}$ ), so that the droplet could sink. Additional insight in the phenomenon can be obtained by numerical simulation. Performed in a linear gradient, the numerical results are found to qualitatively fit the experimental results [Figs. 2(f)-2(j); see Supplemental Material [35] for more details and results].

From both experiment and numerics we conclude that the buoyancy jet is quite strong shortly after the drop is released [Figs. 2(a), 2(f)], then it decreases, so that the Marangoni stress becomes relatively stronger [Figs. 2(b), $2(\mathrm{~g})]$, forming a downwards replenishing flow above the apex of the drop. This local recirculation close to the drop only decreases the Marangoni strength slowly (Pe $\sim 10$, a moderate advection). Sinking deeper, the buoyancy jet becomes so weak until finally it vanishes, and the Marangoni-induced replenishing flow dominates. At this moment [Figs. 2(c), 2(h)], the buoyanct flow stops and the liquid velocity above the drop is entirely downward. Different from the local recirculation, this downward flow brings ethanol-richer liquid to the apex of the drop, decreases the local surface tension, thus increasing the Marangoni flow (especially in the upper half), which in turn brings more ethanol, forming a positive feedback [Figs. 2(d)-2(e), 2(i)-2(j)]. The Marangoni flow consequently strongly increases until it is large enough to pull the droplet up.

The Marangoni velocity $V_{M}$ at the side of the drop and velocity one radius above the drop $V_{R}$ are measured [at positions as indicated in Fig. 2(c)] and plotted in Fig. 3(a). The velocity of the drop $V_{\text {drop }}$ is also plotted as a reference. Indeed, $V_{M}$ slowly decreases while $V_{R}$ is positive (pointing upwards), and $V_{M}$ starts to increase when $V_{R}$ changes direction, entering the positive feedback regime. The Marangoni flow is proportional to the surface tension difference on the drop, $V_{M} \propto \Delta \sigma=$ $2 R\left(\partial \sigma / \partial w_{e}\right)\left(\partial w_{e} / \partial y\right) \approx \partial \sigma / \partial w_{e}\left(w_{e, B}-w_{e, A}\right)$, where $w_{e, A}$ and $w_{e, B}$ are the ethanol fractions of the surrounding mixture at the apex and bottom of the droplet, respectively [Fig. 2(d)]. The drop jumps from regions of ethanol fraction less than $30 \mathrm{wt} \%$, below which, according to Fig. 1(e), the interfacial tension decreases linearly, so that $\partial \sigma / \partial w_{e}$ is a negative constant, thus $V_{M} \propto\left(w_{e, A}-w_{e, B}\right)$. Consider the initial accelerating period where ethanol does not reach the drop's bottom yet, so only $w_{e, A}$ changes. Then $d V_{M} / d t \propto d w_{e, A} / d t$. In the region where the droplet jumps, the concentration of ethanol above the drop can 

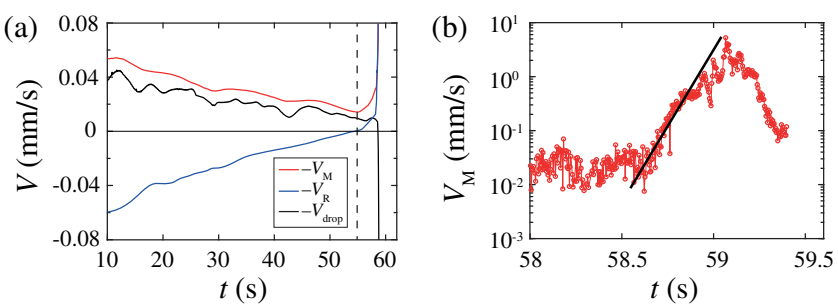

FIG. 3. Measured velocities as a function of time. (a) $V_{M}$ (red), $V_{R}$ (blue) and sinking velocity $V_{\text {drop }}$ (black) during the first jumping cycle. $V_{M}$ and $V_{R}$ are measured at the positions shown in Fig. 2(c). Positive velocity is upward. The absolute value of $V_{M}$, $V_{\text {drop }}$ and $V_{R}$ all decrease. $V_{R}$ vanishes and then changes direction. This leads to the sharp increase of $V_{M}$ around $t \approx 55 \mathrm{~s}$, until it is large enough to pull the droplet up. (b) $V_{M}$ in logarithmic scale as a function of time in the later stage. $V_{M}$ increases exponentially between 58.6 and $59.1 \mathrm{~s}$.

be approximated as linear, $w_{e} \propto y$, so that $d w_{e, A} / d t \propto d y / d t$. The flow field at this moment is induced by the dominating Marangoni flow, so $d y / d t \propto V_{M}$, thus $d V_{M} / d t \propto V_{M}$. This gives rise to an exponential growth of the Marangoni flow:

$$
V_{M} \sim e^{t / \tau}
$$

A zoomed in logarithmic plot of $V_{M}$ is shown in Fig. 3(b). Indeed, $V_{M}$ is confirmed to increase exponentially shortly after it starts to increase. The calculated time constant $\tau$ of the growth is $0.073 \mathrm{~s}$, fitting well with the measured value $0.084 \mathrm{~s}$ (see Supplemental Material [35] for coefficients). Note that the Marangoni flow remarkably increases by more than 2 orders of magnitude within $1 \mathrm{~s}$, accounting for the sudden shooting up.

$V_{M}$ keeps increasing until the drop reaches a higher position where the ethanol fraction $w_{e}>40 \mathrm{wt} \%$ because $\partial \sigma / \partial w_{\mathrm{e}}$ decreases sharply in this region, until it almost vanishes at $w_{e} \approx 60 \mathrm{wt} \%$ [Fig. 1(e)], corresponding to $h \approx 6 \mathrm{~mm}$, which forms the "ceiling" for the jumping because the Marangoni driving force ceases. $V_{M}$ also decreases because when $V_{M}$ reaches its highest value of $\sim 5 \mathrm{~mm} / \mathrm{s}$; the drop's Péclet number is then on the order of 1000. This strong advection tends to homogenize the surrounding liquid [30], leading to an additional decrease of $\partial w_{\mathrm{e}} / \partial y$. This explains the formation of the almost uniform drifted liquid layer [Fig. 2(a)]. For a newly released drop, Marangoni flow during the injection process is responsible for the formation of the uniform layer. Note that when the droplet is sinking, the drifted layer around the drop also decreases in its ethanol concentration (Fig. 2) because of advection and diffusion. This means that the interfacial tension of the droplet is building up, and the deeper it sinks, the more interfacial energy it builds up, so that the droplet has more energy to jump a larger distance. This accumulated interfacial energy is later transformed to momentum by Marangoni flow, in a kind of "avalanche" process.
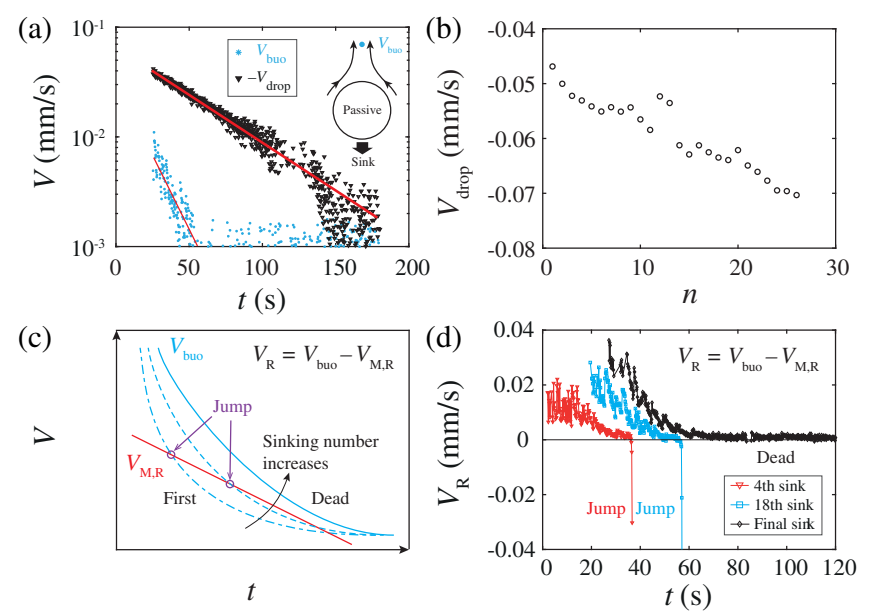

FIG. 4. (a) $V_{\text {drop }}$ and $V_{\text {buo }}$ (measured 1.5 radius above the drop) of a sinking droplet after the addition of surfactant to the bulk liquid. The inset shows the definition of $V_{\text {buo }}$ (b) $V_{\text {drop }}$ at $h=$ $2.5 \mathrm{~mm}$ as a function of the number of the subsequent sinking event. (c) Sketch of the balance between buoyancy jet and Marangoni flow. For later sinking events $n>1, V_{\text {buo }}$ changes from the blue dash-dotted line, to the dashed line and finally to the solid line. Because the last line shown does not intersect with $V_{M, R}$, so $V_{R}=V_{\text {buo }}-V_{M, R}$ does not change direction and the drop does not jump. (d) Measured $V_{R}(t)$ for different sinking cycles. The starting point (highest point) of each cycle is at $t=0$, but the initial stages of the cycles could not be measured because the camera could not record the entire cycle at the necessary frame rate.

The direction of the flow above the droplet, which is determined by the relative strength between buoyancy jet and Marangoni flow, determines whether it jumps or not. Here the buoyancy jet velocity $V_{\text {buo }}$ is the component of $V_{R}$ induced by a sinking passive droplet, i.e., a droplet without Marangoni forces acting on it. To gain more insight into the buoyancy jet here, PIV measurements are performed after the addition of $0.7 \mathrm{mM}$ SDS to suppress the Marangoni flow. It is found that both the sinking velocity $V_{\text {drop }}$ and the buoyancy jet velocity $V_{\text {buo }}$ (measured 1.5 radius above the droplet) decrease exponentially, as shown in Fig. 4(a), with the former one similar to that of a sinking particle [52]. As $V_{M}$ decreases at a much slower rate than $V_{\text {buo }}$ [Fig. 2(a)], at some point the Marangoni flow will dominate, namely, when $V_{\text {drop }}$ is small enough. However, the Marangoni flow is getting weaker by each jump (see Supplemental Material [35]), most likely due to mixing of the surrounding liquid [34], which is enhanced by the jumping itself. Therefore the Marangoni-flow-induced lifting force gets smaller at each jump, so that the sinking velocity increases, as shown in Fig. 4(b), where the sinking velocity at $h=2.5 \mathrm{~mm}$ is plotted against the subsequent number of sinks. Consequently, $V_{\text {buo }}$ increases, and therefore it gets progressively harder for the Marangoni flow to overcome buoyancy. Figure 4(c) shows a sketch of the relative strength between $V_{\text {buo }}$ and $V_{M, R}$ (Marangoni flow induced 
flow 1.5 radius above the drop). For the first sink, $V_{\text {buo }}$ decreases quite fast, and the flow reversal happens when $V_{M, R}$ becomes larger than $V_{\text {buo }}$, so that the droplet jumps. As the number of sinking events increases, $V_{\text {buo }}$ increases and $V_{M, R}$ decreases. Therefore the droplet can sink longer (and also deeper) before it jumps. Finally at some point, $V_{M, R}$ is so weak that $V_{\text {buo }}$ is always dominant: The jumping stops and the droplet falls "dead." We confirm this picture by measuring $V_{R}=V_{\text {buo }}-V_{M, R}$ for a normal sinking droplet without surfactant [Fig. 4(d)]. As expected, $V_{R}$ decreases from a higher value and changes direction at later times as increasing sinking number, until finally no flow reversal is observed, and the droplet falls dead. This multiple bouncing and sudden death behavior is very general because it can be observed for a wide range of droplet sizes, from 0.1 to $5 \mu \mathrm{L}$.

In conclusion, an oil droplet of trans-Anethole released in a vertically stratified ethanol-water mixture is found to bounce repeatedly with ever increasing jumping distance, until finally it falls dead all of a sudden. Marangoni flow and gravity are responsible for this phenomenon: Interfacial energy builds up when the droplet sinks, and a flow reversal above the sinking droplet triggers an exponential growth of the Marangoni flow, leading to the sudden jump. The consequent strong advection decreases the Marangoni stress, enabling the droplet to sink again and then continue the bouncing cycle. The ever decreasing Marangoni flow by each jump is responsible for the droplet's increasing jumping height as well as its sudden death.

The present system can be easily generalized to other liquids, as long as one has a vertically stratified liquid which can generate strong enough Marangoni stress on the droplet. This is supported by our observation of a silicon oil drop in the same stratified fluid that exhibits a similar bouncing behavior. Currently, we are investigating how the other parameters influence this behavior.

Valuable discussions with Chao Sun, Xiaojue Zhu, Guillaume Lajoinie, Huanshu Tan, Yaxing Li, and Luoqin Liu are greatly appreciated. G. Lajoinie also provided valuable technical support. We acknowledge support from the Netherlands Center for Multiscale Catalytic Energy Conversion (MCEC), an NWO Gravitation programme funded by the Ministry of Education, Culture and Science of the government of Netherlands, and ERC-Advanced Grant under Project No. 740479. X. Z. acknowledges the support of the Natural Sciences and Engineering Research Council of Canada (NSERC) and Future Energy Systems (Canada First Research Excellence Fund).

*xuehua.zhang@ualberta.ca

†d.lohse@utwente.nl

[1] C. C. Maass, C. Krüger, S. Herminghaus, and C. Bahr, Swimming droplets, Annu. Rev. Condens. Matter Phys. 7, 171 (2016).
[2] A. J. Demello, Control and detection of chemical reactions in microfluidic systems, Nature (London) 442, 394 (2006).

[3] L. Baraban, F. Bertholle, M. L. M. Salverda, N. Bremond, P. Panizza, J. Baudry, J. A. G. M. de Visser, and J. Bibette, Millifluidic droplet analyser for microbiology, Lab Chip 11, 4057 (2011).

[4] J. Toner and Y. Tu, Flocks, herds, and schools: A quantitative theory of flocking, Phys. Rev. E 58, 4828 (1998).

[5] H. H. Wensink and H. Löwen, Emergent states in dense systems of active rods: from swarming to turbulence, J. Phys. Condens. Matter 24, 464130 (2012).

[6] H. H. Wensink, J. Dunkel, S. Heidenreich, K. Drescher, R. E. Goldstein, H. Löwen, and J. M. Yeomans, Meso-scale turbulence in living fluids, Proc. Natl. Acad. Sci. U.S.A. 109, 14308 (2012).

[7] I. Buttinoni, J. Bialké, F. Kümmel, H. Löwen, C. Bechinger, and T. Speck, Dynamical Clustering and Phase Separation in Suspensions of Self-Propelled Colloidal Particles, Phys. Rev. Lett. 110, 238301 (2013).

[8] J. Bialké, H. Löwen, and T. Speck, Microscopic theory for the phase separation of self-propelled repulsive disks, Europhys. Lett. 103, 30008 (2013).

[9] L. E. Scriven and C. V. Sternling, The Marangoni effects, Nature (London) 187, 186 (1960).

[10] F. Yang, S. Shin, and H. A. Stone, Diffusiophoresis of a charged drop, J. Fluid Mech. 852, 37 (2018).

[11] M. Schmitt and H. Stark, Swimming active droplet: A theoretical analysis, Europhys. Lett. 101, 44008 (2013).

[12] S. Michelin, E. Lauga, and D. Bartolo, Spontaneous autophoretic motion of isotropic particles, Phys. Fluids 25, 061701 (2013).

[13] N. Yoshinaga, K. H. Nagai, Y. Sumino, and H. Kitahata, Drift instability in the motion of a fluid droplet with a chemically reactive surface driven by Marangoni flow, Phys. Rev. E 86, 016108 (2012).

[14] M. M. Hanczyc, T. Toyota, T. Ikegami, N. Packard, and T. Sugawara, Fatty acid chemistry at the oil-water interface: Self-propelled oil droplets, J. Am. Chem. Soc. 129, 9386 (2007).

[15] T. Toyota, N. Maru, M. M. Hanczyc, T. Ikegami, and T. Sugawara, Self-propelled oil droplets consuming "fuel" surfactant, J. Am. Chem. Soc. 131, 5012 (2009).

[16] N. M. Kovalchuk and D. Vollhardt, Marangoni instability and spontaneous non-linear oscillations produced at liquid interfaces by surfactant transfer, Adv. Colloid Interface Sci. 120, 1 (2006).

[17] K. Nagai, Y. Sumino, H. Kitahata, and K. Yoshikawa, Mode selection in the spontaneous motion of an alcohol droplet, Phys. Rev. E 71, 065301(R) (2005).

[18] Y. J. Chen, Y. Nagamine, and K. Yoshikawa, Self-propelled motion of a droplet induced by Marangoni-driven spreading, Phys. Rev. E 80, 016303 (2009).

[19] A. A. Peña and C.A. Miller, Solubilization rates of oils in surfactant solutions and their relationship to mass transport in emulsions, Adv. Colloid Interface Sci. 123-126, 241 (2006).

[20] Z. Izri, M. N. van der Linden, S. Michelin, and O. Dauchot, Self-Propulsion of Pure Water Droplets by Spontaneous Marangoni-Stress-Driven Motion, Phys. Rev. Lett. 113, 248302 (2014). 
[21] H. Tanaka and T. Araki, Spontaneous Double Phase Separation Induced by Rapid Hydrodynamic Coarsening in Two-Dimensional Fluid Mixtures, Phys. Rev. Lett. 81, 389 (1998).

[22] N. Vladimirova, A. Malagoli, and R. Mauri, Diffusiophoresis of two-dimensional liquid droplets in a phaseseparating system, Phys. Rev. E 60, 2037 (1999).

[23] P. Poesio, G. P. Beretta, and T. Thorsen, Dissolution of a Liquid Microdroplet in a Nonideal Liquid-Liquid Mixture far from Thermodynamic Equilibrium, Phys. Rev. Lett. 103, 064501 (2009).

[24] H. Tan, C. Diddens, P. Lv, J. G. M. Kuerten, X. Zhang, and D. Lohse, Evaporation-triggered microdroplet nucleation and the four life phases of an evaporating Ouzo drop, Proc. Natl. Acad. Sci. U.S.A. 113, 8642 (2016).

[25] Y. Li, P. Lv, C. Diddens, H. Tan, H. Wijshoff, M. Versluis, and D. Lohse, Evaporation-Triggered Segregation of Sessile Binary Droplets, Phys. Rev. Lett. 120, 224501 (2018).

[26] H. Kim, K. Muller, O. Shardt, S. Afkhami, and H. A. Stone, Solutal Marangoni flows of miscible liquids drive transport without surface contamination, Nat. Phys. 13, 1105 (2017).

[27] D. M. Sigman, S. L. Jaccard, and G. H. Haug, Polar ocean stratification in a cold climate, Nature (London) 428, 59 (2004).

[28] N. O. Young, J. S. Goldstein, and M. J. Block, The motion of bubbles in a vertical temperature gradient, J. Fluid Mech. 6, 350 (1959).

[29] J. L. Anderson, Colloid transport by interfacial forces, Annu. Rev. Fluid Mech. 21, 61 (1989).

[30] S. Michelin and E. Lauga, Phoretic self-propulsion at finite Péclet numbers, J. Fluid Mech. 747, 572 (2014).

[31] D. Richard, C. Clanet, and D. Quéré, Contact time of a bouncing drop, Nature (London) 417, 811 (2002).

[32] W. Bouwhuis, R. C. A. van der Veen, T. Tran, D. L. Keij, K. G. Winkels, I. R. Peters, D. van der Meer, C. Sun, J. H. Snoeijer, and D. Lohse, Maximal Air Bubble Entrainment at Liquid-Drop Impact, Phys. Rev. Lett. 109, 264501 (2012).

[33] M. Shirota, M. A. J. van Limbeek, C. Sun, A. Prosperetti, and D. Lohse, Dynamic Leidenfrost Effect: Relevant Time and Length Scales, Phys. Rev. Lett. 116, 064501 (2016).

[34] F. Blanchette and A. M. Shapiro, Drops settling in sharp stratification with and without Marangoni effects, Phys. Fluids 24, 042104 (2012).

[35] See Supplemental Material at http://link.aps.org/ supplemental/10.1103/PhysRevLett.122.154502 for more details and videos of the experiment and simulation, which includes Refs. [36-44].

[36] D. Lin, J. R. Leger, M. Kunkel, and P. McCarthy, Onedimensional gradient-index metrology based on ray slope measurements using a bootstrap algorithm, Opt. Eng. 52, 112108 (2013).

[37] W. Thielicke and E. J. Stamhuis, PIVlab-towards userfriendly, affordable and accurate digital Particle Image
Velocimetry in MATLAB, J. Open Res. Software 2, e30 (2014).

[38] W. Thielicke, The flapping flight of birds: Analysis and application, thesis, Rijksuniversiteit Groningen 2014, https://www.rug.nl/research/portal/files/14094714/ Complete_dissertation.pdf.

[39] I. Biró, K. Gábor Szabó, B. Gyüre, I. M. Jánosi, and T. Tél, Power-law decaying oscillations of neutrally buoyant spheres in continuously stratified fluid, Phys. Fluids 20, 051705 (2008).

[40] R. A. Cairncross, P. R. Schunk, T. A. Baer, R. R. Rao, and P. A. Sackinger, A finite element method for free surface flows of incompressible fluids in three dimensions. Part I. Boundary fitted mesh motion, Int. J. Numer. Methods Fluids 33, 375 (2000).

[41] A. N. Brooks and T.J.R. Hughes, Streamline upwind/ Petrov-Galerkin formulations for convection dominated flows with particular emphasis on the incompressible Navier-Stokes equations, Comput. Methods Appl. Mech. Eng. 32, 199 (1982).

[42] S. Chu and A. Prosperetti, Dissolution and growth of a multicomponent drop in an immiscible liquid, J. Fluid Mech. 798, 787 (2016).

[43] Dortmund Data Bank, 2017, http://www.ddbst.com/.

[44] M. Heil and A. L. Hazel, oomph-lib-an object-oriented multi-physics finite-element library, in Fluid-Structure Interaction (Springer, New York, 2006), pp. 19-49.

[45] C. R. Torres, H. Hanazaki, J. Ochoa, J. Castillo, and M. van Woert, Flow past a sphere moving vertically in a stratified diffusive fluid, J. Fluid Mech. 417, 211 (2000).

[46] H. Hanazaki, K. Kashimoto, and T. Okamura, Jets generated by a sphere moving vertically in a stratified fluid, J. Fluid Mech. 638, 173 (2009).

[47] K. Y. Yick, C. R. Torres, T. Peacock, and R. Stocker, Enhanced drag of a sphere settling in a stratified fluid at small Reynolds numbers, J. Fluid Mech. 632, 49 (2009).

[48] H. Hanazaki, K. Konishi, and T. Okamura, Schmidt-number effects on the flow past a sphere moving vertically in a stratified diffusive fluid, Phys. Fluids 21, 026602 (2009).

[49] I. Eames, D. Gobby, and S. B. Dalziel, Fluid displacement by Stokes flow past a spherical droplet, J. Fluid Mech. 485, 67 (2003).

[50] K. Katija and J. O. Dabiri, A viscosity-enhanced mechanism for biogenic ocean mixing, Nature (London) 460, 624 (2009).

[51] J. C. Nawroth and J. O. Dabiri, Induced drift by a selfpropelled swimmer at intermediate Reynolds numbers, Phys. Fluids 26, 091108 (2014).

[52] Y. Zvirin and R.S. Chadwick, Settling of an axially symmetric body in a viscous stratified fluid, Int. J. Multiphase Flow 1, 743 (1975). 